\title{
Labels Available on Branded Men's Readymade Garments
}

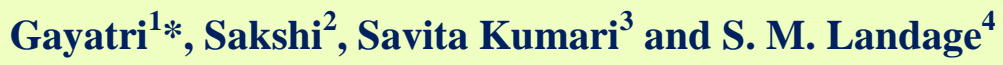 \\ ${ }^{1}$ Department of Textile and Apparel Designing, College of Community Science, DRPCAU, \\ Pusa-848125, Bihar, India \\ ${ }^{2}$ Department of Clothing \& Textiles, GBPUA\&T, Pantnagar-263145, Uttarakhand, India \\ ${ }^{3} I C D S$ Project, K V K, Hariharpur, Vaishali, DRPCAU, Pusa, Bihar, India \\ ${ }^{4}$ D. K. T. E. Society's, Textile and Engineering Institute, \\ Ickalkaranji-416115, Maharashtra, India \\ *Corresponding author
}

\section{A B S T R A C T}

Earlier fabric was made up entirely of one fiber type and people preferred to wear tailor made garments. But due to industrialization, advancement in technologies various type of fabric and readymade garments are available in market. Due to this, consumer need and

Keywords

Label on garment, Branded showroom, Men's readymade, Brand for male clothing

\section{Article Info}

Accepted:

22 July 2020

Available Online:

10 August 2020 demand continuously change with the time and availability of the products. In regarding this, the wearing style of people changed and now they prefer to wear readymade garments. Nowadays, buying of fabric and selection of readymade garments and their care are not as easy as in the days of our grandmother. In such situation and more in the absence of labels, information about product is given by the seller which may not be reliable. Thus, consumers are unable to judge the actual quality, cost and care of the readymade garment. In this situation, labelling works as tool for assuring customer regarding the quality, cost and care of readymade garments. Keeping in view these points the study was conducted to collect information about labels which are presented on readymade men's wear. A self structure interview schedule was prepared. Fifteen owner of branded showroom from Rudrapur, Uttarakhand were purposively selected as sample size. Interview schedule consist two part i.e., general information and specific information. Study showed that all readymade men's wear categories i.e., casual, formal, winter, groom and sport wear had labels pertaining information of size, brand labels, company name, bar code and care. It was also found that maximum number of labels, presented in inside the side seam and at centre back of the garment. Temporary label of tag type was present on all the readymade garments.

\section{Introduction}

The apparel and textiles sector has been equipped with better tool and techniques. Therefore, change have been so numerous and so rapid that every few years there are new names for weaves, colour, finishes and the new synthetic fabric and garment (Gayatri and Sakshi, 2014) ${ }^{(1)}$. So consumers are unable to judge the quality and cost of garments 
available in the market for consumers. Beside this, at present an intelligent consumer is not satisfied with attractive appearance of the material only. They look for other quality to when they buy their cloths. They want to know the nature of the fiber, if it is serviceable, whether its colour is fast or fade and if it will launder well, if it shrinks and so, how much and most important of all, whether it is worth the money spend on it. In such situation labels provide information about garment's quality, guarantee about colour etc. and also people now a day go and buy garment directly from the showroom at reasonable price because readymade garment have advantages compare to tailor made garments.

In India there is no textile labelling act exist at present. But under Textile (Consumer Protection) Regulation, 1988 there is mention of labels for indigenously manufactured and imported textiles, namely tops, yarn and cloth. Federal Trade Commission (FTC) had prepared guide to help to comply with federal labelling requirements for textile, wool and fur products (Gayatri and Sakshi, 2014) ${ }^{(2)}$. The law requires that most textile and wool products have a label listing: the fiber content, the country of origin, and the identity of the manufacturer or another business responsible for marketing or handling the item. It also provide the information regarding mechanics of labelling, label placement and attachment for hosiery and other product sold in packages.

Information provided on the label is usually correct and regulated by law. So there was need to check that which kind of label present on readymade garments. So keeping in view the present study was planned to collect information related labels with the objectives:- To collect information about labels available on branded readymade men's wear.

\section{Materials and Methods}

The fifteen branded showrooms were selected as sample for the present study, which were involved in the sale of readymade men's wear. The data was collected by personal interview method. A self structure interview schedule was formulated with the help of subject matter specialist.

Interview schedule had two parts. Part A of interview schedule dealt with general information of the branded showrooms which included name of the showroom, year of establishment, address, type of ownership, type of retail format, financial aid to start showroom. The part B of the schedule consisted of questions related to types and the position of labels present on different garments such as informative labels, care labels, placements of labels, and type of attachment of the labels to the garment. The garments were selected for checking labels had to fulfill the following criteria; it should have maximum number labels compared to other garments of same category. The statistical measures used for the analysis were frequency and percentage.

\section{Results and Discussion}

\section{General information of showroom owner}

Table 1 gives information about distribution of branded showroom according to type of ownership, type of retail format and source of financial aid to start the showroom. It is evident from the Table that maximum i.e., 73.33 per cent of the showroom owners were sole proprietors and rest $(26.67 \%)$ had partnership. Sixty per cent of the showrooms had franchisee type of retail format. Maximum showroom owners started their showroom by investing their own money. Only twenty per cent of the showroom owners took loan for starting the showrooms. 
Table 2 gives information regarding sale assortment of different branded showrooms. It is clear from table that casual shirts, jeans and t- shirts were produced and sold by maximum number of showrooms. It can be inferred from table that only two showrooms sold sport wear and wedding suits. It is evident from table that maximum branded sold all formal and winter wear except coat in winter wear.

\section{Labels on casual wear}

Table 3 gives data pertaining to labels present on branded men's casual wears which included shirts, jeans, bermuda shorts and tshirts.

\section{Shirts}

All the shirts had labels pertaining to size, brand name, company name, bar code, care labeling regarding washing, ironing. Labels were placed inside the side seam, temporary labels of tag type and permanent labels of loop type were present on all the shirts.

\section{Jeans}

All type of jeans irrespective of brand had labels related to size, brand name, company name, bar code and care label including washing, bleaching, ironing and drying. Maximum (83.33\%) labels were placed at top centre back of the jeans that at waistline. Tag type temporary labels were attached in all the jeans while square and rectangular stitch types of labels were found in jeans in maximum showrooms.

\section{Bermuda shorts}

All the showroom selling bermuda shorts had labels pertaining to size, brand name, company name, bar code and care labelling regarding washing and ironing irrespective of their brand. Tag type of labels was attached in all the bermuda shorts of the branded showroom.

\section{T - Shirt}

Labels pertaining to size, brand name, company name, bar code, care labelling regarding washing, ironing and drying were seen in all the t- shirt irrespective of brand. All the t-shirts had labels which were placed at inside side seam.

\section{Labels on men's formal wear}

Table 4 gives information regarding labels present in branded men's formal wear, which included trousers, blazers, suits and shirts. It is clear from the Table that irrespective of all types of formal wear, labels pertaining to size, brand name, company name, bar code and also for care labels were present on all the garments irrespective of their showrooms. Temporary type of tag labels were attached on all the men's formal wear irrespective of their age group category.

\section{Labels on men's winter clothing}

Table 5 gives information related to labels present on branded men's winter clothing, which include namely; Sweater both full and half, coat, waistcoat, cardigan, sweatshirts, blazers and jacket.

\section{Full sweater}

Labels related to size, brand name, company name, bar code were seen in all the full sweaters irrespective of brand. Tag type temporary label was presented on all the full sweaters.

\section{Half sweater}

In case of half sweater size, brand name, company name, bar code were presented on 
all the half sweaters. Care labels pertaining to washing, bleaching, ironing and drying were present on maximum half sweaters i. e., $90 \%$. Tags, were the types of labels temporary attached to all the half sweaters.

\section{Coat}

All the branded coats had labels related to country of origin, size, brand name, company name, bar code, care labelling regarding drycleaning and temporary labels of tag type.

\section{Waistcoat}

All the waistcoats had labels related to size, brand name, company name, bar code and care labels regarding washing, bleaching and ironing irrespective of brand.

\section{Cardigan}

Labels pertaining to size, brand name, company name, bar code were presented on all the cardigan irrespective of brand. Care label related to washing, bleaching, ironing and drying were seen on maximum cardigans irrespective of brand i. e., $83.33 \%$.

\section{Sweatshirts}

All the sweatshirt had labels related to fiber content, size, brand name, company name, bar code and care regarding washing, bleaching, ironing and drying.

\section{Blazers}

In case of blazers, labels pertaining to size, brand name, company name, bar code and care labels regarding dry-cleaning and temporary labels of tag type were seen on all the blazers irrespective of brand.

\section{Jackets}

All the branded jackets had labels pertaining to size, brand name, company name, bar code and temporary labels of tag type.

Table.1 Distribution of branded showrooms according to general information

\begin{tabular}{|r|l|c|c|}
\hline S. No. & \multicolumn{1}{|c|}{ Variables } & Frequency & Per cent \\
\hline 1 & \multicolumn{1}{|c|}{ Type of ownership } \\
\hline A & Sole proprietorship/ family business & 11 & 73.33 \\
\hline B & Partnership & 04 & 26.67 \\
\hline 2 & \multicolumn{1}{|c|}{ Type of Retail format } & \\
\hline A & Specialty store & - & 33.33 \\
\hline B & Departmental store & 05 & -60.00 \\
\hline C & Franchisee & 01 & 6.67 \\
\hline D & Exclusive & & \\
\hline 3 & Source of financial aid & 03 & 20.00 \\
\hline A & Loan & 12 & 80.00 \\
\hline B & Self & & \\
\hline
\end{tabular}


Table.2 Distribution of branded showrooms according to men' readymade garments present in their sale assortment

\begin{tabular}{|c|c|c|c|}
\hline S.No. & & & Name of Brand \\
\hline 1. & \multicolumn{3}{|c|}{$\begin{array}{l}\text { Name of Garment Name of Brand } \\
\text { Men's Casual Clothing }\end{array}$} \\
\hline A & \multicolumn{2}{|c|}{ Shirts } & $\begin{array}{l}\text { Reebok Store, Pepe Jeans, Black Berrys, The Raymond, Charlie Outlow, } \\
\text { Levi's, Lee, Allen Solly, Vivacity, Cantabil, Spykar, Liverpool, Koutons. }\end{array}$ \\
\hline B & \multicolumn{2}{|l|}{ Jeans } & $\begin{array}{l}\text { Reebok store, Pepe Jeans, The Raymond, Charlie Outlow, Levi's, Lee, Allen } \\
\text { Solly, Vivacity, Cantabil. Spykar, Liverpool, Koutons. }\end{array}$ \\
\hline $\mathbf{C}$ & \multicolumn{2}{|c|}{ Bermuda shorts } & $\begin{array}{l}\text { Reebok store, Pepe Jeans, Charlie Outlow, Levi’s, Lee, Cantabil, Spykar, } \\
\text { Liverpool, Koutons, Adidas. }\end{array}$ \\
\hline D & \multicolumn{2}{|l|}{ T-Shirt } & $\begin{array}{l}\text { Reebok Store, Pepe Jeans, Charlie Outlow, Levi's, Lee, Allen Solly, Vivacity, } \\
\text { Adidas, Cantabil, Spykar, Liverpool, Koutons. }\end{array}$ \\
\hline 2. & \multicolumn{3}{|c|}{ Men's Formal Wear } \\
\hline A & \multicolumn{2}{|c|}{ Trousers } & $\begin{array}{l}\text { Pepe Jeans, Black Berrys, The Raymond, Charlie outlow, Swayamvar, Lee, } \\
\text { Allen Solly, Vivacity, Cantabil, Koutons. }\end{array}$ \\
\hline B & \multicolumn{2}{|l|}{ Blazers } & $\begin{array}{l}\text { Black Berrys, The Raymond, Charlie outlow, Swayamvar, Allen Solly, } \\
\text { Vivacity, Cantabil, Koutons. }\end{array}$ \\
\hline $\mathbf{C}$ & \multicolumn{2}{|l|}{ Suits } & Black berrys, The Raymond, Swayamvar, Allen Solly, Cantabil, Koutons. \\
\hline D & \multicolumn{2}{|l|}{ Shirts } & $\begin{array}{l}\text { Black Berrys, The Raymond, Levi's, Swayamvar, Lee, Allen Solly, Vivacity, } \\
\text { Cantabil, Koutons. }\end{array}$ \\
\hline 3. & \multicolumn{3}{|c|}{ Men's Winter Clothing } \\
\hline \multirow[t]{2}{*}{ A } & \multirow[b]{2}{*}{ Sweater } & Full & $\begin{array}{l}\text { Reebok store, Pepe Jeans, The Raymond, Charlie Outlow, Levi's, Lee, } \\
\text { Vivacity, Adidas, Cantabil, Spykar, Koutons. }\end{array}$ \\
\hline & & Half & $\begin{array}{l}\text { Reebok Store, Pepe Jeans, The Raymond, Charlie outlow, Levi's, Lee, Adidas, } \\
\text { Cantabil, Spykar, Koutons. }\end{array}$ \\
\hline B & \multicolumn{2}{|l|}{ Coat } & Levi’s, Koutons. \\
\hline $\mathbf{C}$ & \multicolumn{2}{|l|}{ Waistcoat } & Pepe jeans, Charlie outlow, Koutons \\
\hline D & \multicolumn{2}{|c|}{ Cardigans } & The Raymond, Charlie outlow, Vivacity, Adidas, Spykar, Koutons. \\
\hline $\mathbf{E}$ & \multicolumn{2}{|c|}{ Sweat shirts } & $\begin{array}{l}\text { Reebok Store, Pepe Jeans, Levi’s, Lee, Vivacity, Cantabil, Catabil, Spykar, } \\
\text { Koutons. }\end{array}$ \\
\hline $\mathbf{F}$ & \multicolumn{2}{|l|}{ Blazers } & $\begin{array}{l}\text { Reebok Store, The Raymond, Allen solly, Cantabil, Spykar, Liverpool, } \\
\text { Koutons. }\end{array}$ \\
\hline G & \multicolumn{2}{|l|}{ Jacket } & $\begin{array}{l}\text { Reebok Store, The Raymond, Charlie Outlow, Levi's, Lee, Allen solly, Adidas, } \\
\text { Cantabil, Spykar, Liverpool, Koutons. }\end{array}$ \\
\hline 4. & \multicolumn{3}{|r|}{ 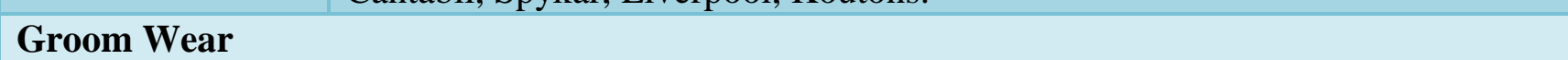 } \\
\hline A & \multicolumn{2}{|c|}{ Wedding Suit } & Black Berrys, Swayamvar. \\
\hline B & \multicolumn{2}{|c|}{ Sherwani } & Swayamvar \\
\hline 5. & \multicolumn{2}{|c|}{ Men's Nightwear } & None of the branded showroom sold Men's night wears. \\
\hline 6. & \multirow{2}{*}{\multicolumn{2}{|c|}{$\begin{array}{l}\text { Men's Sportswear } \\
\text { Tracksuit }\end{array}$}} & \\
\hline a. & & & Reebok and Adidas. \\
\hline b. & \multicolumn{2}{|c|}{ Jogging Shorts } & Reebok and Adidas. \\
\hline
\end{tabular}


Table.3 Distribution of branded showroom for men's casual wears according to label information present on them

\begin{tabular}{|c|c|c|c|c|c|c|c|c|c|c|c|c|}
\hline \multirow[t]{2}{*}{$\begin{array}{l}\text { S. } \\
\text { No. }\end{array}$} & \multirow{2}{*}{\multicolumn{4}{|c|}{ Attributes }} & \multicolumn{2}{|c|}{$\begin{array}{l}\text { Shirts } \\
\mathbf{N}=13\end{array}$} & \multicolumn{2}{|c|}{$\begin{array}{l}\text { Jeans } \\
\mathrm{N}=12\end{array}$} & \multicolumn{2}{|c|}{$\begin{array}{l}\text { Bermuda } \\
\text { shorts } N=10\end{array}$} & \multicolumn{2}{|c|}{$\begin{array}{l}\text { T- Shirt } \\
\mathrm{N}=12\end{array}$} \\
\hline & & & & & $\mathbf{F}$ & $\%$ & $\mathbf{F}$ & $\%$ & $\mathbf{F}$ & $\%$ & $\mathbf{F}$ & $\%$ \\
\hline 1 & \multicolumn{4}{|c|}{ Fiber content } & 04 & 30 & 06 & 50.00 & 01 & 10.00 & 04 & 33.33 \\
\hline 2 & \multicolumn{4}{|c|}{ Country of origin } & 06 & 46.15 & 04 & 33.33 & 04 & 40.00 & 07 & 58.33 \\
\hline 3 & \multicolumn{4}{|c|}{ Size } & 13 & 100 & 12 & 100 & 10 & 100 & 12 & 100 \\
\hline 4 & \multicolumn{4}{|c|}{ Brand name } & 13 & 100 & 12 & 100 & 10 & 100 & 12 & 100 \\
\hline 5 & \multicolumn{4}{|c|}{ Company name } & 13 & 100 & 12 & 100 & 10 & 100 & 12 & 100 \\
\hline 6 & \multicolumn{4}{|c|}{ Certification labels } & 02 & 15.38 & 0 & 0 & 0 & 0 & 01 & 8.33 \\
\hline 7 & \multicolumn{4}{|c|}{ Bar code } & 13 & 100 & 12 & 100 & 10 & 100 & 12 & 100 \\
\hline \multirow[t]{5}{*}{8} & \multirow{5}{*}{ U. } & \multicolumn{3}{|c|}{ Washing } & 13 & 100 & 12 & 100 & 10 & 100 & 12 & 100 \\
\hline & & \multicolumn{3}{|c|}{ Bleaching } & 12 & 92.31 & 12 & 100 & 9 & 90.00 & 11 & 91.67 \\
\hline & & \multicolumn{3}{|c|}{ Ironing } & 13 & 100 & 12 & 100 & 10 & 100 & 12 & 100 \\
\hline & & \multicolumn{3}{|c|}{ Dry cleaning } & 0 & 0 & 0 & 0 & 0 & 0 & 0 & 0 \\
\hline & & \multicolumn{3}{|c|}{ Drying } & 12 & 92.31 & 12 & 100 & 9 & 90.00 & 12 & 100 \\
\hline \multirow[t]{4}{*}{9} & \multirow{4}{*}{ 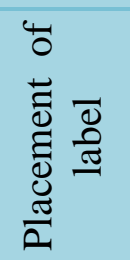 } & \multirow{2}{*}{\multicolumn{3}{|c|}{$\begin{array}{l}\text { Collar band } \\
\text { Top center back of } \\
\text { the garment }\end{array}$}} & 2 & 15.38 & 0 & 0 & 0 & 0 & 0 & 0 \\
\hline & & & & & 11 & 84.62 & 10 & 83.33 & 9 & 90.00 & 11 & 91.67 \\
\hline & & \multirow{2}{*}{\multicolumn{2}{|c|}{\begin{tabular}{l|l}
$\begin{array}{l}\text { Side } \\
\text { seam }\end{array}$ & $\mathrm{I}$ \\
$\mathrm{C}$
\end{tabular}}} & & 13 & 100 & 0 & 0 & 0 & 0 & 12 & 100 \\
\hline & & & & side & 0 & 0 & 0 & 0 & 02 & 20.00 & 02 & 16.67 \\
\hline \multirow[t]{10}{*}{10} & \multirow{10}{*}{ 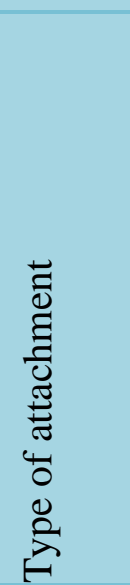 } & \multirow{4}{*}{ 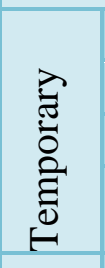 } & \multicolumn{2}{|c|}{ On wrapper/package } & 0 & 0 & 0 & 0 & 0 & 0 & $\mathrm{O}$ & 0 \\
\hline & & & \multirow{2}{*}{\multicolumn{2}{|c|}{$\begin{array}{l}\text { Tag } \\
\text { Removable ticket }\end{array}$}} & 13 & 100 & 12 & 100 & 10 & 100 & 12 & 100 \\
\hline & & & & & 0 & 0 & 0 & 0 & 0 & 0 & 0 & 0 \\
\hline & & & Pampl & & 02 & 15.38 & 01 & 8.33 & 01 & 10.00 & 0 & 0 \\
\hline & & & Printe & & 04 & 30.77 & 0 & 0 & 02 & 20.00 & 04 & 33.33 \\
\hline & & $\frac{2}{8}$ & & Loop & 13 & 100 & 05 & 41.67 & 04 & 40.00 & 11 & 91.67 \\
\hline & & 寻 & ச छิ & Square & 05 & 38.46 & 06 & 50.00 & 01 & 10.00 & 0 & 0 \\
\hline & & एँ & 吾? & Rectangular & 07 & 53.85 & 06 & 50.00 & 09 & 90.00 & 06 & 50.00 \\
\hline & & 气్ & & Mitered & 01 & 7.69 & 0 & 0 & 01 & 10.00 & 00 & 25.00 \\
\hline & & $\begin{array}{c}\overline{0} \\
2\end{array}$ & Embrc & idered & 03 & 23.08 & 03 & 25.00 & 03 & 30.00 & 02 & 16.67 \\
\hline
\end{tabular}


Table.4 Distribution of branded showroom for men's formal wears according to label information present on them

\begin{tabular}{|c|c|c|c|c|c|c|c|c|c|c|c|c|}
\hline \multirow[t]{2}{*}{$\begin{array}{l}\text { S. } \\
\text { No. }\end{array}$} & \multirow{2}{*}{\multicolumn{4}{|c|}{ Attributes }} & \multicolumn{2}{|c|}{$\begin{array}{c}\text { Trousers } \\
\text { N=8 }\end{array}$} & \multicolumn{2}{|c|}{$\begin{array}{c}\text { Blazers } \\
N=10\end{array}$} & \multicolumn{2}{|c|}{$\begin{array}{l}\text { Suits } \\
\quad \mathrm{N}=6\end{array}$} & \multicolumn{2}{|c|}{$\begin{array}{l}\text { Shirts } \\
\quad \mathrm{N}=9\end{array}$} \\
\hline & & & & & $\mathbf{F}$ & $\%$ & $\mathbf{F}$ & $\%$ & $\mathbf{F}$ & $\%$ & $\mathbf{F}$ & $\%$ \\
\hline 1 & \multicolumn{4}{|c|}{ Fiber content } & 01 & 12.5 & 05 & 50.00 & 02 & 33.33 & 03 & 33.33 \\
\hline 2 & \multicolumn{4}{|c|}{ Country of origin } & 02 & 25.00 & 01 & 10.00 & 0 & 0 & 05 & 55.56 \\
\hline 3 & \multicolumn{4}{|c|}{ Size } & 08 & 100 & 10 & 100 & 06 & 100 & 09 & 100 \\
\hline 4 & \multicolumn{4}{|c|}{ Brand name } & 08 & 100 & 10 & 100 & 06 & 100 & 09 & 100 \\
\hline 5 & \multicolumn{4}{|c|}{ Company name } & 08 & 100 & 10 & 100 & 06 & 100 & 09 & 100 \\
\hline 6 & \multicolumn{4}{|c|}{ Certification labels } & 0 & 0 & 0 & 0 & 0 & 0 & 01 & 11.11 \\
\hline 7 & \multicolumn{4}{|c|}{ Bar code } & 08 & 100 & 10 & 100 & 06 & 100 & 09 & 100 \\
\hline \multirow[t]{5}{*}{8} & \multicolumn{4}{|c|}{\begin{tabular}{|l|l|} 
Washing \\
\end{tabular}} & 07 & 87.50 & 0 & 0 & 0 & 0 & 07 & 77.78 \\
\hline & \multicolumn{4}{|c|}{$\stackrel{\infty}{\Xi}$ Bleaching } & 07 & 87.50 & 0 & 0 & 0 & 0 & 07 & 77.78 \\
\hline & \multirow{3}{*}{ שี } & \multirow{2}{*}{\multicolumn{3}{|c|}{$\begin{array}{l}\text { Ironing } \\
\text { Drv cleaning }\end{array}$}} & 08 & 100 & 0 & 0 & 0 & 0 & 07 & 77.78 \\
\hline & & & & & 0 & 0 & 10 & 100 & 06 & 100 & 02 & 22.22 \\
\hline & & \multicolumn{3}{|c|}{ Drying } & 08 & 100 & 0 & 0 & 0 & 0 & 07 & 77.78 \\
\hline \multirow[t]{4}{*}{9} & \multirow{4}{*}{$\begin{array}{l}\bar{\Xi} \\
\bar{\Xi} \\
\bar{\Xi} \\
\frac{\sigma}{\pi} \\
\frac{\tilde{\sigma}}{\alpha}\end{array}$} & \multicolumn{3}{|c|}{ Collar band } & 0 & 0 & 0 & 0 & 0 & 0 & 0 & 0 \\
\hline & & \multicolumn{3}{|c|}{ Top center back of the garment } & 03 & 37.5 & 04 & 40.00 & 01 & 16.67 & 04 & 44.44 \\
\hline & & \multirow{2}{*}{$\begin{array}{l}\text { Side } \\
\text { seam }\end{array}$} & \multicolumn{2}{|c|}{ Inside } & 0 & 0 & 0 & 0 & 0 & 0 & 09 & 100 \\
\hline & & & Outside & & 0 & 0 & 0 & 0 & 0 & 0 & 0 & 0 \\
\hline \multirow[t]{10}{*}{10} & \multirow{10}{*}{ 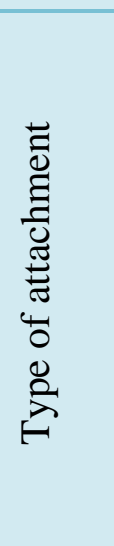 } & \multirow{4}{*}{ 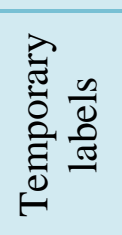 } & \multirow{2}{*}{\multicolumn{2}{|c|}{$\begin{array}{l}\text { On wrapper/package } \\
\text { Tag }\end{array}$}} & 01 & 12.5 & 0 & 0 & 0 & 0 & 0 & 0 \\
\hline & & & & & 08 & 100 & 10 & 100 & 06 & 100 & 09 & 100 \\
\hline & & & Remor & le ticket & 0 & 0 & 0 & 0 & 0 & 0 & 0 & 0 \\
\hline & & & Pamph & & 0 & 0 & 01 & 10.00 & 0 & 0 & 0 & 0 \\
\hline & & & Printec & & 01 & 12.5 & 04 & 40.00 & 0 & 0 & 02 & 22.22 \\
\hline & & & & Loop & 02 & 25 & 04 & 40.00 & 01 & 16.67 & 06 & 66.67 \\
\hline & & $\stackrel{\infty}{ \pm} \frac{\infty}{0}$ & $\stackrel{0}{0}$ & Square & 04 & 50 & 07 & 70.00 & 03 & 50 & 02 & 22.22 \\
\hline & & है & 吾方 & Rectangular & 04 & 50 & 07 & 70.00 & 05 & 83.33 & 07 & 77.78 \\
\hline & & & & Mitered & 0 & 0 & 0 & 0 & 0 & 0 & 02 & 22.22 \\
\hline & & & Embro & & 01 & 12.5 & 01 & 10.00 & 0 & 0 & 0 & 0 \\
\hline
\end{tabular}


Table.5 Distribution of branded showroom for men's winter wears according to label information present on them

\begin{tabular}{|c|c|c|c|c|c|c|c|c|c|c|c|c|c|c|c|c|c|c|}
\hline \multirow{3}{*}{$\begin{array}{l}\text { S. } \\
\text { No } \\
\text {. }\end{array}$} & \multirow{3}{*}{\multicolumn{3}{|c|}{ Attributes }} & \multicolumn{4}{|c|}{ Sweater } & \multirow{2}{*}{\multicolumn{2}{|c|}{$\begin{array}{l}\text { Coat } \\
\mathrm{N}=2\end{array}$}} & \multirow{2}{*}{\multicolumn{2}{|c|}{$\begin{array}{c}\text { Waist } \\
\text { coat } \\
\mathbf{N}=3\end{array}$}} & \multirow{2}{*}{$\begin{array}{c}\text { Card } \\
\text { i-gan } \\
N=6\end{array}$} & \multirow{2}{*}{\multicolumn{2}{|c|}{$\begin{array}{c}\text { Sweat } \\
\text { shirts } \\
\mathrm{N}=9\end{array}$}} & \multirow{2}{*}{\multicolumn{2}{|c|}{$\begin{array}{c}\text { Blazer } \\
N=7\end{array}$}} & \multirow{2}{*}{\multicolumn{2}{|c|}{$\begin{array}{c}\text { Jacket } \\
\mathrm{N}=11\end{array}$}} \\
\hline & & & & \multicolumn{2}{|c|}{$\begin{array}{c}\text { Full } \\
\mathbf{N}=11\end{array}$} & \multicolumn{2}{|c|}{$\begin{array}{c}\text { Half } \\
\mathbf{N}=10\end{array}$} & & & & & & & & & & & \\
\hline & & & & $\mathbf{F}$ & $\%$ & $\mathbf{F}$ & $\%$ & $\mathbf{F}$ & $\%$ & $\mathbf{F}$ & $\%$ & F \% & & $\%$ & & $\%$ & $\mathbf{F}$ & $\%$ \\
\hline 1 & \multicolumn{3}{|c|}{ Fiber content } & 9 & 81.82 & 9 & 90.00 & 0 & 0 & 1 & 33.33 & 583.33 & 9 & 100 & 5 & 71.43 & 5 & 45.45 \\
\hline 2 & \multicolumn{3}{|c|}{ Country of origin } & 4 & 36.36 & 4 & 40.0 & 2 & 100 & 0 & 0 & 233.33 & & 22.22 & 2 & 28.57 & 4 & 36.36 \\
\hline 3 & \multicolumn{3}{|c|}{ Size } & 11 & 100 & 10 & 100 & 2 & 100 & 3 & 100 & $\begin{array}{ll}6 & 100\end{array}$ & 9 & 100 & 7 & 100 & 11 & 100 \\
\hline 4 & \multicolumn{3}{|c|}{ Brand name } & 11 & 100 & 10 & 100 & 2 & 100 & 3 & 100 & $\begin{array}{ll}6 & 100\end{array}$ & 9 & 100 & 7 & 100 & 11 & 100 \\
\hline 5 & \multicolumn{3}{|c|}{ Company name } & 11 & 100 & 10 & 100 & 2 & 100 & 3 & 100 & $\begin{array}{ll}6 & 100\end{array}$ & 9 & 100 & 7 & 100 & 11 & 100 \\
\hline 6 & \multicolumn{3}{|c|}{ Certification labels } & 0 & 0 & 0 & 0 & 0 & 0 & 0 & 0 & \begin{tabular}{l|l}
0 & 0
\end{tabular} & 0 & 0 & 0 & 0 & 1 & 9.09 \\
\hline 7 & \multicolumn{3}{|c|}{ Bar code } & 11 & 100 & 10 & 100 & 0 & 0 & 3 & 100 & $\begin{array}{ll}6 & 100\end{array}$ & 9 & 100 & 7 & 100 & 11 & 100 \\
\hline \multirow[t]{6}{*}{8} & \multirow{6}{*}{ 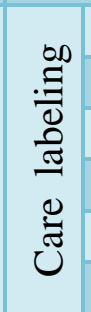 } & \multicolumn{2}{|c|}{ Washing } & 10 & 90.91 & 9 & 90.00 & 0 & 0 & 3 & 100 & 583.33 & 9 & 100 & 0 & 0 & 2 & 18.18 \\
\hline & & \multicolumn{2}{|c|}{ Bleaching } & 10 & 90.91 & 9 & 90.0 & 0 & 0 & 3 & 100 & 583.33 & 9 & 100 & 0 & 0 & 1 & 9.09 \\
\hline & & \multicolumn{2}{|c|}{ Ironing } & 10 & 90.91 & 9 & 90.0 & 0 & 0 & 3 & 100 & 583.33 & 9 & 100 & 0 & 0 & 1 & 9.09 \\
\hline & & \multicolumn{2}{|c|}{ Dry cleaning } & 1 & 9.09 & 1 & 10.00 & 2 & 100 & 1 & 33.33 & 116.67 & 1 & 11.11 & 7 & 100 & 10 & 90.91 \\
\hline & & Dryil & & 10 & 90.91 & 9 & 90.00 & 0 & 0 & 2 & 66.67 & 583.33 & 9 & 100 & 0 & 0 & 1 & 9.09 \\
\hline & & Warr & ning (twist) & 4 & 36.36 & 4 & 40.0 & 0 & 0 & 1 & 33.33 & 116.67 & 1 & 11.11 & 0 & 0 & 0 & 0 \\
\hline 9 & & Colla & ar band & 0 & 0 & 0 & 0 & 0 & 0 & 0 & 0 & \begin{tabular}{l|l}
0 & 0
\end{tabular} & 0 & 0 & 0 & 0 & 0 & 0 \\
\hline & $\begin{array}{l}\overline{0} \\
\text { हृ }\end{array}$ & $\begin{array}{l}\text { Top } \\
\text { Of tl }\end{array}$ & $\begin{array}{l}\text { center back } \\
\text { the garment }\end{array}$ & 6 & 54.54 & 5 & 50.00 & 0 & 0 & 0 & 0 & 466.67 & 5 & 55.55 & 2 & 28.57 & 5 & 45.45 \\
\hline & $\frac{\pi}{2}$ & Side & Inside & 10 & 90.91 & 8 & 80.00 & 0 & 0 & 1 & 33.33 & 683.33 & & 55.55 & 0 & 0 & 5 & 45.45 \\
\hline & & seam & Outside & 0 & 0 & 2 & 20.00 & 0 & 0 & 0 & 0 & 0 & 0 & 0 & 0 & 0 & 2 & 18.18 \\
\hline 10 & & $\widehat{\vec{z}}$ & $\begin{array}{l}\text { On } \\
\text { wrapper/package }\end{array}$ & 0 & 0 & 0 & 0 & 0 & 0 & 0 & 0 & 0 & 0 & 0 & 0 & 0 & 0 & 0 \\
\hline & $\exists$ & 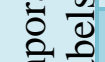 & Tag & 11 & 100 & 10 & 100 & 2 & 100 & 3 & 100 & $\begin{array}{ll}6 & 100\end{array}$ & 9 & 100 & 7 & 100 & 11 & 100 \\
\hline & $\overline{\mathbb{Q}}$ & $\overline{0} \Xi$ & Removable ticket & 0 & 0 & 0 & 0 & 0 & 0 & 0 & 0 & \begin{tabular}{l|l}
0 & 0
\end{tabular} & 0 & 0 & 0 & 0 & 0 & 0 \\
\hline & चี & & Pamphlet & 0 & 0 & 0 & 0 & 0 & 0 & 1 & 33.33 & 0 & 0 & 0 & 1 & 14.28 & 0 & 0 \\
\hline & $\bar{\Xi}$ & & Printed & 4 & 36.36 & 2 & 20.00 & 1 & 50.00 & 1 & 33.33 & \begin{tabular}{l|l}
116.67
\end{tabular} & 3 & 33.33 & 1 & 14.28 & 4 & 36.36 \\
\hline & to & & Loop & 7 & 63.63 & 6 & 60.00 & 1 & 50.00 & 1 & 33.33 & \begin{tabular}{|l|l|}
6 & 100
\end{tabular} & & 77.77 & 2 & 28.57 & 9 & 81.82 \\
\hline & $\sum_{\equiv}^{0}$ & $\bar{\Xi}$ & ల్ Ðี Square & 2 & 18.18 & 2 & 20.00 & 2 & 100 & 1 & 33.33 & $\begin{array}{ll}0 & 0\end{array}$ & & 11.11 & 3 & 42.86 & 7 & 63.63 \\
\hline & 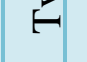 & 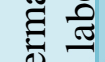 & \& Rectangular & 4 & 36.36 & 3 & 30.00 & 2 & 100 & 0 & 0 & 0 & & 22.22 & 4 & 57.14 & 4 & 36.36 \\
\hline & & 2 & Mitered & 3 & 27.27 & 2 & 20.00 & 0 & 0 & 0 & 0 & 350.00 & & 11.11 & 0 & 0 & 0 & 0 \\
\hline & & & Embroidered & 3 & 27.27 & 4 & 40.00 & 0 & 0 & 1 & 33.33 & 116.67 & & 11.11 & 0 & 0 & 1 & 9.09 \\
\hline
\end{tabular}


Table.6 Distribution of branded showroom for men's groom and sport wears according to label information present on them

\begin{tabular}{|c|c|c|c|c|c|c|c|c|c|c|c|c|}
\hline \multirow[t]{2}{*}{$\begin{array}{l}\text { S. } \\
\text { No. }\end{array}$} & \multirow{2}{*}{\multicolumn{4}{|c|}{ Attributes }} & \multicolumn{2}{|c|}{$\begin{array}{l}\text { Wedding } \\
\text { suit } \quad \mathrm{N}=2\end{array}$} & \multicolumn{2}{|c|}{$\begin{array}{l}\text { Sherwani } \\
\mathrm{N}=1\end{array}$} & \multicolumn{2}{|c|}{$\begin{array}{l}\text { Track suits } \\
\mathrm{N}=2\end{array}$} & \multicolumn{2}{|c|}{$\begin{array}{l}\text { Jogging } \\
\text { shorts N=2 }\end{array}$} \\
\hline & & & & & $\mathbf{F}$ & $\%$ & $\mathbf{F}$ & $\%$ & $\mathbf{F}$ & $\%$ & $\mathbf{F}$ & $\%$ \\
\hline 1 & \multirow{2}{*}{\multicolumn{4}{|c|}{ Fiber content }} & 0 & 0 & 01 & 100 & 01 & 50.00 & 01 & 50.00 \\
\hline 2 & Country of origin & & & & 0 & 0 & 0 & 0 & 01 & 50.00 & 01 & 50.00 \\
\hline 3 & \multicolumn{4}{|c|}{ Size } & 02 & 100 & 01 & 100 & 02 & 100 & 02 & 100 \\
\hline 4 & \multicolumn{4}{|c|}{ Brand name } & 02 & 100 & 01 & 100 & 02 & 100 & 02 & 100 \\
\hline 5 & \multicolumn{4}{|c|}{ Company name } & 02 & 100 & 01 & 100 & 02 & 100 & 02 & 100 \\
\hline 6 & \multicolumn{4}{|c|}{ Certification labels } & 0 & 0 & 0 & 0 & 0 & 0 & 0 & 0 \\
\hline 7 & \multicolumn{4}{|c|}{ Bar code } & 02 & 100 & 01 & 100 & 02 & 100 & 02 & 100 \\
\hline \multirow[t]{5}{*}{8} & \multirow{5}{*}{ 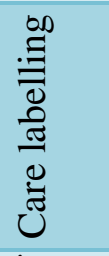 } & \multicolumn{3}{|c|}{ Washing } & 0 & 0 & 0 & 0 & 02 & 100 & 02 & 100 \\
\hline & & \multirow{2}{*}{\multicolumn{3}{|c|}{$\begin{array}{l}\text { Bleaching } \\
\text { Ironing }\end{array}$}} & 0 & 0 & 0 & 0 & 01 & 50.00 & 0 & 0 \\
\hline & & \multirow{2}{*}{\multicolumn{2}{|c|}{$\begin{array}{l}\text { Ironing } \\
\text { Drv cleaning }\end{array}$}} & & 0 & 0 & 0 & 0 & 02 & 100 & 02 & 100 \\
\hline & & \multicolumn{2}{|c|}{ Dry cleaning } & & 02 & 100 & 01 & 100 & 0 & 0 & 0 & 0 \\
\hline & & \multicolumn{3}{|c|}{ Drying } & 0 & 0 & 0 & 0 & 02 & 100 & 02 & 100 \\
\hline \multirow[t]{4}{*}{9} & \multirow{4}{*}{ 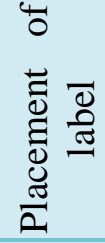 } & & 0 & 0 & 0 & 0 & 0 & 0 & 0 & 0 \\
\hline & & \multicolumn{3}{|c|}{$\begin{array}{l}\text { Top center back } \\
\text { of the garment }\end{array}$} & 0 & 0 & 01 & 100 & 02 & 100 & 02 & 100 \\
\hline & & \multicolumn{3}{|c|}{$\underset{0}{0}$ Inside } & 0 & 0 & 0 & 0 & 02 & 100 & 02 & 100 \\
\hline & & $\ddot{n}$ & Out & side & 0 & 0 & 0 & 0 & 0 & 0 & 0 & 0 \\
\hline \multirow[t]{10}{*}{10} & \multirow{10}{*}{ 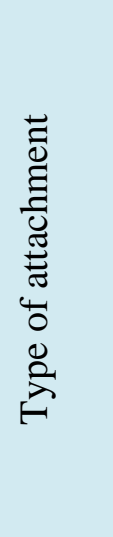 } & \multirow{4}{*}{ 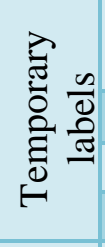 } & \multicolumn{2}{|c|}{$\begin{array}{l}\text { On wrapper/ } \\
\text { package }\end{array}$} & 0 & 0 & 0 & 0 & 0 & 0 & 0 & 0 \\
\hline & & & $\operatorname{tag}$ & & 02 & 100 & 01 & 100 & 02 & 100 & 02 & 100 \\
\hline & & & Ren & novable ticket & 0 & 0 & 0 & 0 & 0 & 0 & 0 & 0 \\
\hline & & & Pam & phlet & 0 & 0 & 0 & 0 & 0 & 0 & 0 & 0 \\
\hline & & & Prin & & 0 & 0 & 0 & 0 & 01 & 50.00 & 01 & 50.00 \\
\hline & & $\vec{\nabla}$ & & Loop & 0 & 0 & 01 & 100 & 01 & 50.00 & 02 & 100 \\
\hline & & $\underset{\pi}{\infty}$ & లే & Square & 02 & 100 & 0 & 0 & 0 & 0 & 0 & 0 \\
\hline & & ह్ర త్తి & & Rectangular & 02 & 100 & 01 & 100 & 0 & 0 & 0 & 0 \\
\hline & & & & Mitred & 0 & 0 & 0 & 0 & 0 & 0 & 0 & 0 \\
\hline & & & Emb & roidered & 0 & 0 & 0 & 0 & 01 & 50.00 & 0 & 0 \\
\hline
\end{tabular}

*Where: $\mathrm{F}=$ Frequency, $\%=$ Per cent

$* \mathrm{~N}=$ Total number of showrooms

\section{Labels on men's groom and sport wear}

Table 6 gives data pertaining to labels present on branded men's groom wear i.e., wedding suit, sherwani and men's sport wear i. e., Track suits and Jogging shorts.

\section{Wedding suits}

All branded wedding suits had labels related to size, brand name, company name, bar code and also care labels which related to dry-cleaning. Tag was temporary label attached to all wedding suits.

\section{Sherwani}

Sherwani sold by Swayamvar had labels related to fiber content, size, brand name, company name, bar code and care label regarding dry- 
cleaning. Tags were temporary labels attached to sherwani.

\section{Track suits}

All track suit had labels related to size, brand name, company name, bar code and care label regarding washing, ironing and drying. Labels were placed at top centre back and inside side seam of the track suits. All track suits had temporary tag type labels.

\section{Jogging shorts}

All the jogging shorts had labels related to size, brand name, company name, bar code and care label regarding washing, ironing and drying. Tag was temporary type label attached to all jogging shorts. Loop type permanent label attached inside of side seam of all jogging shorts.

\section{Labels on men's night wear}

None of branded showrooms sold night wear in their sale assortment. Night wear includes pajamas and night suits.

In conclusion, the study show that casual shirts, jeans and $\mathrm{T}$ - shirt sold by maximum number of showroom. And also found the maximum showrooms sold all formal and winter wear except coat in winter which was sold by only two showrooms. General information showed that maximum i.e., 73.33 per cent branded showroom owners were sole proprietors. Sixty per cent of the showrooms were had franchisee type of retail outlets, maximum started their showroom by investing their own money.

Some other significant findings of the study were that all readymade men's wear categories i.e., casual, formal, winter, groom, night and sport wear had labels depicting information of size, brand name, company name, bar code and care including washing, ironing, drying and dry cleaning. Maximum numbers of labels were found in inside the side seam and at centre back of the garment i.e., neck in upper garments and waist in lower garments. Temporary labels of tag type were present on all the readymade garment. Information regarding fibre content and country of origin were not present in maximum no. of garment which were mandatory as FTC regulation.

\section{References}

Gayatri and Sakshi. A Comparative study: labels on readymade garments with standards. The Journal of Rural and Agricultural Research. 2014; 14 (2): 9396.

Gayatri and Sakshi. Knowledge assessment of consumers on readymade garments. Progressive Research: An International Journal. 2014; 09 (2): 272-275.

Gayatri and Sakshi. Wardrobe of male teaching professionals. The Journal of Rural and Agricultural Research. 2014; 14 (2): 7881.

Gayatri, Sakshi and Virk, B. Consumer awareness regarding label present on ready-made garments. The Journal of Rural and Agricultural Research. 2015; 15 (1): 65-67.

Gill, P., Grewal, N. and Lata, P. Trends in textile labeling. Textile Trends. 2005; 48 (1): 41-46.

http://www.ehow.com/list_6082830_lawsclothing-label-requirements. html. Retrieved on September, 3, 2010.

\section{How to cite this article:}

Gayatri, Sakshi, Savita Kumari and Landage, S. M. 2020. Labels Available on Branded Men's Readymade Garments. Int.J.Curr.Microbiol.App.Sci. 9(08): 2823-2832. doi: https://doi.org/10.20546/ijcmas.2020.908.317 